\title{
SEARCH FOR LEPTOQUARKS AT HERA-I
}

\author{
C. HELEBRANT \\ On behalf of the H1 Collaboration \\ Deutsches Elektronen-Synchrotron DESY \\ Notkestrasse 85 \\ 22607 Hamburg, Germany \\ E-mail: christian.helebrant@desy.de
}

\begin{abstract}
A search for scalar and vector leptoquarks coupling to first generation fermions is performed using the $e^{+} p$ and $e^{-} p$ scattering data collected by the $\mathrm{H} 1$ experiment between 1994 and 2000 [1]. The data correspond to a total integrated luminosity of $117 \mathrm{pb}^{-1}$. No evidence for the direct or indirect production of such particles is found in data samples with a large transverse momentum final state electron or with large missing transverse momentum. Constraints on leptoquark models are established. For leptoquark couplings of electromagnetic strength, leptoquarks with masses up to $275-325 \mathrm{GeV}$ are ruled out.
\end{abstract}

\section{Introduction}

Leptoquarks (LQs) are color triplet bosons which couple directly to a lepton and a parton. They appear in many unifying theories beyond the Standard Model (SM). The phenomenological model used in this analysis was proposed by Buchmüller, Rückl and Wyler (BRW) [2]. We use here the nomenclature of [3] to label the various scalar or vector LQ types.

14 types of first generation $\mathrm{LQs}^{\mathrm{a}}$ are differentiated by their spin, weak isospin and chirality. They can also be classified by their fermion number $F$. All of the LQs can decay into $e^{ \pm} q$, and four of them have a second decay channel into $\nu q$. For the latter ones the branching ratios $\beta_{e}$ and $\beta_{\nu}$ are both fixed to 0.5 . The dimensionless parameter $\lambda$, which defines the coupling at the lepton-quark-LQ vertex, and the LQ mass $M$ are free parameters in this model.

The ep collider HERA offers the unique possibility of a resonant production of LQs in the $s$-channel via fusion of the incoming lepton with a quark

a.e. LQs coupling only to first generation leptons and quarks 
from the proton at a mass equal to the center-of-mass energy of the leptonquark system. Alternatively, LQs could be exchanged in the $u$-channel. The LQ decays lead to final states similar to those of deep-inelastic scattering (DIS) neutral current (NC) and charged current (CC) interactions at very high $Q^{2}$, the negative four-momentum transfer squared. Thus, only a statistical detection is possible. The sensitive quantities are the reconstructed LQ mass and the spectrum $d \sigma / d y$, where the inelasticity variable $y$ is defined as $y=Q^{2} / M^{2}$.

\section{Data Analysis}

The search for LQs is performed using the full $e^{+} p$ and $e^{-} p$ data accumulated by H1 between 1994 and 2000 corresponding to a total integrated luminosity of $117 \mathrm{pb}^{-1}$. The kinematic domain is limited to $Q^{2}>2500 \mathrm{GeV}^{2}$ and $0.1<y<0.9$. The mass spectra measured for NC- and CC-like events are compared in Fig. 1(left) with the SM predictions, obtained using a Monte-Carlo (MC) calculation. In all cases the data are well described by the SM prediction. Since no evidence for LQ production is observed in either the $\mathrm{NC}$ or $\mathrm{CC}$ data samples, the data are used to set constraints on LQs which couple to first generation fermions.

For the limit analysis, the data are studied in bins in the $M-y$ plane. A weight proportional to the signal-to-background ratio is applied to each bin. The number of background events in each bin is obtained from the SM MC calculations. To estimate the LQ signal, an event re-weighting technique is applied to the same SM MC events [1]. The limits are determined from a statistical analysis which uses the method of fractional event counting [4].

\section{Limit Results}

For LQs with $F=0$, the upper limits on the coupling obtained at 95\% CL are shown as a function of the LQ mass in Fig. 1(right), for scalar and vector LQs respectively. For masses above $\sim 270 \mathrm{GeV}$, these bounds improve by a factor of about 3 the limits obtained in [5] from the analysis of $e^{+} p$ data at $\sqrt{s_{e p}}=300 \mathrm{GeV}$ only. Constraints corresponding to $F=2 \mathrm{LQs}$ are also shown in Fig. 1(right) which extend those in [6] beyond the kinematic limit. For a coupling of electromagnetic strength $\alpha_{\mathrm{em}}\left(\lambda=\sqrt{4 \pi \alpha_{\mathrm{em}}}=0.3\right)$ this analysis rules out LQ masses below 275 to $325 \mathrm{GeV}$, depending on the LQ type.

Fig. 2(left) summarises the constraints on the $\tilde{S}_{1 / 2, L}$ and on the $S_{0, L}$ obtained by H1, by the OPAL and L3 experiments at LEP [7], and by the 

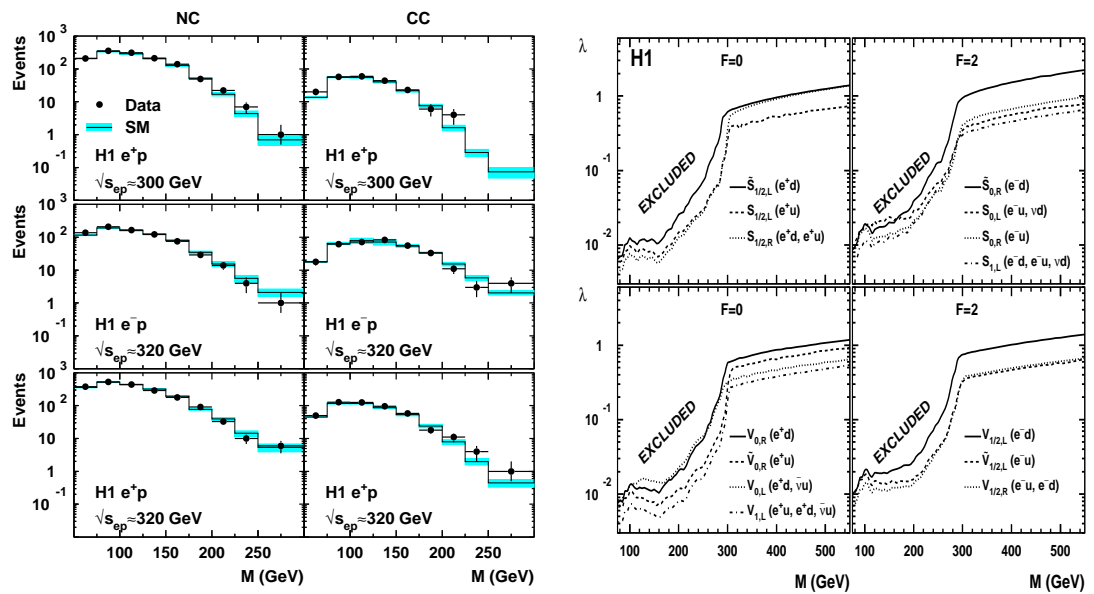

Figure 1. Left: Mass spectra for the NC and CC DIS selected events, together with the corresponding SM expectations. The shaded bands indicate the $\pm 1 \sigma$ uncertainty on the SM expectation. Right: Exclusion limits for the 14 LQs described by the BRW model. The limits are expressed at 95\% CL on the coupling $\lambda$ as a function of the leptoquark mass.

D0 experiment at the Tevatron [8]. Especially in the domain of resonant LQ production, the limits obtained by this analysis extend beyond the domains excluded by the other experiments. The limits at high mass values show good agreement with those obtained in a contact interaction analysis [9].

Beyond the BRW ansatz, generic LQ models can also be considered. In generic LQ models other LQ decay modes are allowed such that the branching ratios $\beta_{e}$ and $\beta_{\nu}$ are free parameters. Mass dependent constraints on the LQ branching ratios can then be set for a given value of $\lambda$. Fig 2(right) shows the domain of the $\beta_{e}-M\left(\beta_{\nu}-M\right)$ plane excluded by the NC (CC) analysis for different values of the coupling $\lambda$, both for a vector and a scalar LQ. For small couplings $\lambda=0.06$ and $\lambda=0.03$ the resulting combined bound is largely independent of the individual values of $\beta_{e}$ and $\beta_{\nu}$. For a coupling $\lambda=0.3$ and high $\beta_{\nu}$ the limit extends to high mass values above the kinematic limit of resonant LQ production. A smooth transition is observed between limits driven by resonant production and limits driven by contact interactions. The domain excluded by the D0 experiment at the Tevatron [8] is also shown. For $\lambda$ greater than $\sim 0.06$, the H1 limits on scalar LQs extend considerably beyond the region ruled out by the D0 experiment. 

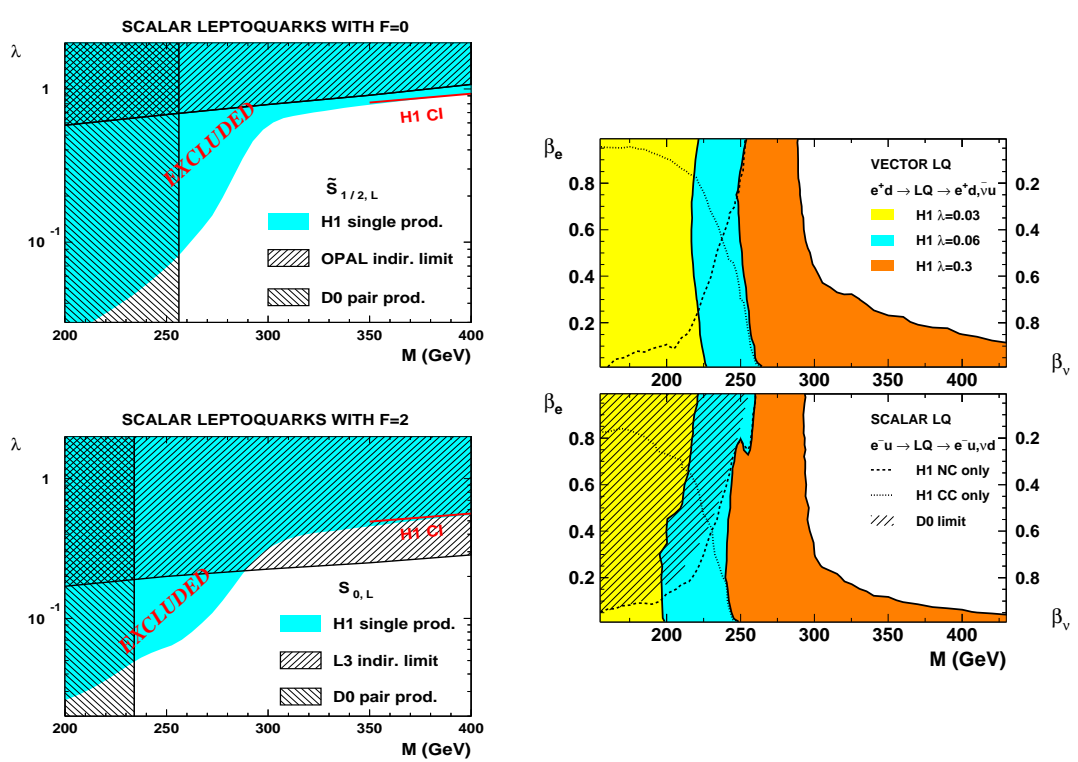

Figure 2. Left: Exclusion limits at $95 \% \mathrm{CL}$ on the coupling $\lambda$ as a function of the LQ mass for $\tilde{S}_{1 / 2, L}$ (top) and $S_{0, L}$ (bottom) in the framework of the BRW model. Constraints on LQs with masses above $350 \mathrm{GeV}$, obtained from the $\mathrm{H} 1$ contact interaction (H1 CI) analysis [9], are shown in the rightmost part of the figures. Right: Domains ruled out by the combination of the $\mathrm{NC}$ and $\mathrm{CC}$ analyses for three values of the coupling $\lambda$. The branching ratios $\beta_{e}$ and $\beta_{\nu}\left(=1-\beta_{e}\right)$ are shown on the left and right axes respectively. The regions on the left of the full curves are excluded at 95\% CL.

\section{References}

1. A. Aktas et al. [H1 Collaboration], Phys. Lett. B 629 (2005) 9

2. W. Buchmüller, R. Rückl and D. Wyler, Phys. Lett. B 191 (1987) 442 [Erratum-ibid. B 448 (1999) 320].

3. A. Djouadi, T. Köhler, M. Spira and J. Tutas, Z. Phys. C 46 (1990) 679.

4. P. Bock, [hep-ex/0405072].

5. C. Adloff et al. [H1 Collaboration], Eur. Phys. J. C 11 (1999) 447 [Erratumibid. C 14 (1999) 553]

6. C. Adloff et al. [H1 Collaboration], Phys. Lett. B. 523 (2001) 234

7. G. Abbiendi et al. [OPAL Collaboration], Eur. Phys. J. C 6 (1999) 1

8. V. M. Abazov [D0 Collaboration], Phys. Rev. D 71 (2005) 071104

9. C. Adloff et al. [H1 Collaboration], Phys. Lett. B 568 (2003) 35

10. C. Adloff et al. [H1 Collaboration], Eur. Phys. J. C 30 (2003) 1 